\title{
ESTRATEGIAS DE SALUD ELECTRÓNICA EN LA REGIÓN DE LAS AMÉRICAS: SITUACIÓN ACTUAL Y PERSPECTIVAS
}

\author{
ELECTRONIC HEALTH STRATEGIES IN THE AMERICAS: CURRENT SITUATION AND \\ PERSPECTIVES
}

\author{
Marcelo D'Agostino ${ }^{1, a}$ \\ Organización Panamericana de la Salud, Organización Mundial de la Salud. \\ MagIster en Gestión del Conocimiento, director del Departamento de Gestión del Conocimiento, Bioética e Investigación, OPS/OMS \\ Recibido: 07-04-15; Aprobado: 22-04-15
}

\begin{abstract}
RESUMEN
El objetivo principal de la Estrategia y Plan de Acción Sobre eSalud (2012-2017) es contribuir al desarrollo sostenible de los sistemas de salud de los estados miembros. Con su adopción se busca mejorar el acceso a los servicios de salud y su calidad a través de la utilización de las tecnologías de la información y de las comunicaciones (TIC), la implementación de programas de alfabetización digital y el acceso a información de calidad que permita avanzar hacia sociedades más informadas, equitativas, competitivas y democráticas. La OPS/OMS considera que sociedades con acceso libre y equitativo a la información sobre salud debe ser un derecho fundamental de las personas. El acceso a información, intercambio de conocimientos y uso de las tecnologías de la información y la comunicación en el sector de la salud sigue creciendo y está impulsando cambios significativos en la manera en que la población interactúa con los servicios de salud y entre sí misma en las redes sociales y mediante el uso de dispositivos móviles (mSalud). Esta sociedad hiperconectada, o sociedad de la información, nos trae nuevos desafíos y oportunidades relacionadas con el uso de datos masivos (Big data) que nos obliga a repensar nuestra relaciones con la realidad y la forma tradicional de manejo de información en salud.
\end{abstract}

Palabras clave: Tecnología de la información; Tecnología biomédica; Informática médica (fuente: DeCS BIREME).

\begin{abstract}
The main objective of the Strategy and Plan of Action on eHealth (2012-2017) is to contribute to sustainable development of health systems of member states. Its adoption aims to improve quality and access to health services through the use of information and communication technologies (ICT), the implementation of digital literacy programs and access to quality information to advance towards more informed, equitable, competitive and democratic societies. PAHO/WHO considers that in society, free and equal access to health information should be a fundamental right of individuals. Access to information, knowledge sharing and use of information and communication technology in the health sector continues to grow and is driving significant changes in the way people interact with health services and among themselves in social networks and through the use of mobile devices (mHealth). This hyper-connected society, or information society, brings new challenges and opportunities related to the use of massive data (Big Data) that forces us to rethink our relationship with reality and the traditional ways of managing health information.
\end{abstract}

Key words: Information technology; Biomedical technology; Medical informatics (source: MeSH NLM).

\section{INTRODUCCIÓN}

Según el análisis de situación descrito en la Estrategia y Plan de Acción Sobre eSalud de la OPS/OMS, el acceso a las tecnologías de la información y la comunicación no es universal, y muchos países y poblaciones de la región disponen de manera desigual de estas herramientas ${ }^{(1)}$. Un estudio realizado en el 2010 por la Unión Internacional de Telecomunicaciones (UIT), muestra que para la región de las Américas la media de altas de línea fija por cada 100 habitantes es de 17,15 , y de $83,3 \%$ en el caso de líneas móviles. En cuanto a la proporción de hogares con una computadora, la media es de $24,2 \%$ mientras que la media de hogares con acceso a Internet es de 13,3\% siendo $25,2 \%$ la media de usuarios de Internet por cada 100 habitantes ${ }^{(2)}$. Más recientemente, la UIT publicó en 2014 nuevas estadísticas en las que se muestra que para las Américas, la media de altas de línea móvil por cada 100 habitantes fue de 108,5\%. Específicamente, con respecto a la media de altas de línea móvil, un total de 27 países, el $63 \%$ de los países que aparecen en esta estadística, superan la barrera de las 100 suscripciones por cada 100 habitantes.

El propósito de la Estrategia y Plan de acción Sobre eSalud (2012-2017) es contribuir al desarrollo sostenible de los sistemas de salud de los estados miembros, incluida la salud pública veterinaria. Con su adopción 
se busca mejorar el acceso a los servicios de salud y su calidad, gracias a la utilización de las tecnologías de la información y de las comunicaciones (TIC), la formación en alfabetización digital ${ }^{(3)}$ y TIC, el acceso a información basada en pruebas científicas y formación continua y la implementación de diversos métodos, permite avanzar hacia sociedades más informadas, equitativas, competitivas y democráticas. Se destaca que en este tipo de sociedades, el acceso a la información sobre salud es un derecho fundamental de las personas.

\section{AVANCES}

La implementación de la estrategia de eSalud de la OPS se viene dando de manera integrada y bajo estrategias o normativas nacionales, o por componentes específicos de: registro médico electrónico (o historia clínica electrónica), telesalud (incluida la telemedicina), mSalud (o salud por dispositivos móviles), eLearning (incluida la formación o aprendizaje a distancia), educación continua en tecnologías de la información y la comunicación bajo el concepto de "alfabetización digital", y estandarización e interoperabilidad. Si bien todos los países, sin excepción, tienen grandes avances en la implementación de tecnologías de información en salud, los países con los que la OPS ha venido avanzando en estrategias nacionales de eSalud son: Argentina, Brasil, Chile, Costa Rica, Republica Dominicana, Guatemala, Jamaica, México, Panamá, Paraguay, Perú y Trinidad y Tobago.

Es importante recordar y reforzar siempre los beneficios que traen la implementación de estrategias nacionales de eSalud presentados y aprobados por todos los estados miembros de la OPS y que se enmarcan en tres grandes componentes de la estrategia regional: innovación, aspectos sociosanitarios y en la economía de cualquier país: a) Desde el punto de vista de la innovación: la eSalud permite transformar los procedimientos de trabajo y mejorar tanto las comunicaciones como la interacción y la gestión del riesgo, así como la seguridad del paciente. La toma de decisiones clínicas fundamentada en evidencia científica, generalmente aumenta la seguridad del paciente. b) Desde el punto de vista sociosanitario: la tecnología aplicada a los procedimientos sanitarios (atención médica y listas de espera, entre otros) contribuye a mejorar la calidad de vida de los pacientes y de la población. Los servicios de salud se caracterizarán por ser más personalizados, integrados y continuos. Al mismo tiempo, estos nuevos servicios permiten superar las limitaciones geográficas y temporales en cuanto a lapsos de espera y acceso a los profesionales de salud y c) Desde el punto de vista económico: las ventajas de aplicar la tecnología a la salud mejoran la eficiencia en el uso del tiempo y los recursos, aumentan los insumos para la toma de decisiones complejas y permiten la asignación de prioridades fundamentadas en pruebas científicas.

Con el fin de debatir, generar y analizar ideas y reflexionar sobre el presente y el futuro del uso de TIC en salud, el Departamento de Gestión del Conocimiento, Bioética e Investigación de la OPS/OMS, convocó a profesionales involucrados en temas de eSalud para desarrollar una serie de diálogos virtuales que abordaron el presente y el futuro de la eSalud en las Américas y el mundo ${ }^{(4)}$. El resultado de estos debates produjeron una importante publicación electrónica llamada "Conversaciones sobre eSalud" que se encuentra disponible en el sitio web de eSalud de la OPS/OMS: http://www.paho.org/ict4health. Esta publicación representa un gran avance para la estrategia y plan de acción de eSalud de la OPS, ya que explora formas de implementar los mecanismos regionales con acceso libre y equitativo a la información y el intercambio de conocimientos, entre otros aspectos. A través de sus 12 capítulos, "Conversaciones sobre eSalud" incorpora la opinión de especialistas, lecturas recomendadas y experiencias exitosas en eSalud. La obra presenta las conclusiones del proyecto, incluye entrevistas con los autores de las conversaciones y presenta un modelo conceptual sobre el cual se basa en trabajo de la OPS/OMS en eSalud (Figura 1).

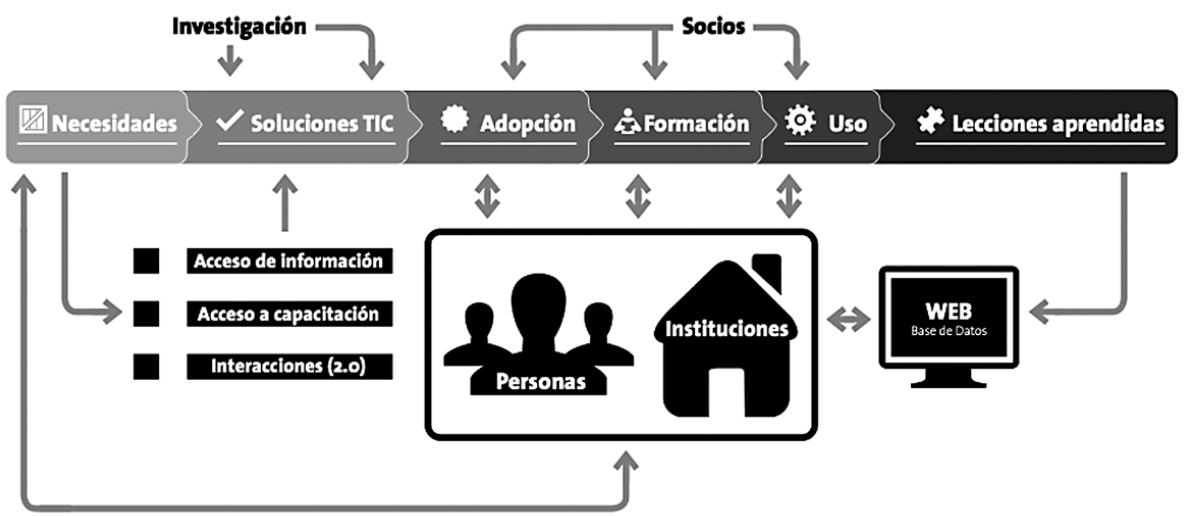

Figura 1.

Conversaciones sobre eSalud. Fuente: OPS/OMS 
Conjuntamente con la Organización Mundial de la Salud y la Unión Internacional de Telecomunicaciones, se desarrolló una caja de herramientas o eHealth toolkit para proporcionar un marco y un método para el desarrollo de una visión nacional de eSalud, un plan de acción y un seguimiento. Es un set de recursos estratégicos que puede ser aplicado por todos los gobiernos que están desarrollando, o comienzan a desarrollar, una estrategia nacional de salud, sea cual sea su nivel actual de avance en proyectos de eSalud ${ }^{(5)}$.

\section{DESAFÍOS}

En el 2011 destacamos tres importantes desafíos que aún siguen siendo validos $\mathrm{y}$, por ello, es importante revisitarlos para poder contextualizar los desafíos más específicos relacionados con la adopción de las TIC en Salud.

En la región de las Américas subsisten tres desafíos importantes en el sector de la salud: a). Acceso limitado a los servicios de salud de amplios segmentos poblacionales debido a las diferencias en los niveles económico, cultural, geográfico y étnico; b). Superposición de perfiles epidemiológicos que obligan a adoptar de manera simultánea varias estrategias sanitarias para hacer frente a las enfermedades transmisibles y a las no transmisibles (estas últimas han aumentado debido al envejecimiento de la población), y c). Insuficiencia de infraestructura, así como de recursos humanos y presupuestarios.

En párrafos siguientes el foco está exclusivamente relacionado con la adopción de las TIC en salud a través de una visión general, ya que cada país enfrenta problemas específicos que corresponden a sus realidades y necesidades, las que enfrentan de acuerdo con sus prioridades y en el marco de sus políticas y regulaciones. Los países enfrentan, y seguirán enfrentando, desafíos relacionados con la adopción de tecnologías de información, ya que las estas se desarrollan a un ritmo mayor que la capacidad que tienen estos países, sus instituciones y las personas en adoptarlas o adaptarlas, y esto nos obliga a repensar nuestra relación con la realidad que nos impone la sociedad de la información.

Un gran desafío general que se presenta con el tema de las TIC, es que mucha de las acciones claves necesarias para su adopción y uso por el bien de la salud, no se limitan ni se encuentran exclusivamente bajo el ámbito de decisiones del sistema de salud y, en la gran mayoría de los casos, se encuentran sujetos a decisiones del sector privado, o bien, junto al sector privado bajo las normas de las entidades nacionales de regulación. Por ejemplo, los temas de conectividad, redes telefónicas, fijas y móviles, y todos los aspectos relacionados con una mejor infraestructura tecnológica son temas que se plantean generalmente como resultado de acciones basadas en otras prioridades y proyectos de Gobiernos y no por mejoras a los sistemas de salud, que si bien se benefician con esto, no son generalmente los que imponen las prioridades de inversiones en temas de infraestructura tecnológica y conectividad en los países.

Un segundo gran desafío, ya en el ámbito de la salud, que enfrentan los países de nuestra región, y del mundo, es el tema de interoperabilidad. Y si bien en el pasado solo se entendía a nivel técnico y dentro del ámbito de los ingenieros de sistemas, sin embargo y poco a poco, el tema se ha instalado en las discusiones y agendas políticas y estratégicas que traen, o deberían traer, resultados y acciones en los ámbitos normativos ${ }^{(6)}$. Sin interoperabilidad real los países corren un altísimo riesgo de hacer fuertes inversiones de recursos, financieros y humanos, que luego tendrán un impacto negativo en los sistemas de salud. Uno de los problemas mas grandes es que la falta de interoperabilidad no se percibe fácilmente si no se tiene una estrategia nacional de eSalud que cuente con una arquitectura de información y telecomunicaciones que permita visualizar la aplicación de las TIC para la salud pública en un periodo no menor a diez años.

Un tercer gran desafío esta relacionado con los procesos de alfabetización digital, entendiéndose a la "alfabetización" como un proceso y no como un "estado". Como se mencionó anteriormente, debido al casi inmanejable ritmo de desarrollo de nuevas tecnologías de información, junto al casi inmanejable e incalculable volumen de información que se va incorporando a cada segundo en la web, es imprescindible desarrollar capacidades en las personas para que puedan desarrollarse funcionalmente, en sus ámbitos profesionales y privados, dentro de la sociedad de la información. Esto repercutirá positivamente en las instituciones en donde se desarrollan y, a su vez, en el trabajo que estas desarrollan por la salud de las personas. Aquí es importante considerar un aspecto relacionado con el ciclo de la responsabilidad que genera tener acceso a la información y al conocimiento especializado en salud que muchos trabajadores de la salud publica tenemos. Para que exista información de calidad accesible y visible tenemos que compartir lo que sabemos y lo que aprendemos diariamente para que aquellos que tienen menos acceso al conocimiento, así como menos infraestructura tecnológicas, se beneficien de los que sí lo tienen. Este ciclo de responsabilidad profesional se ve reflejado en la Figura 2. 


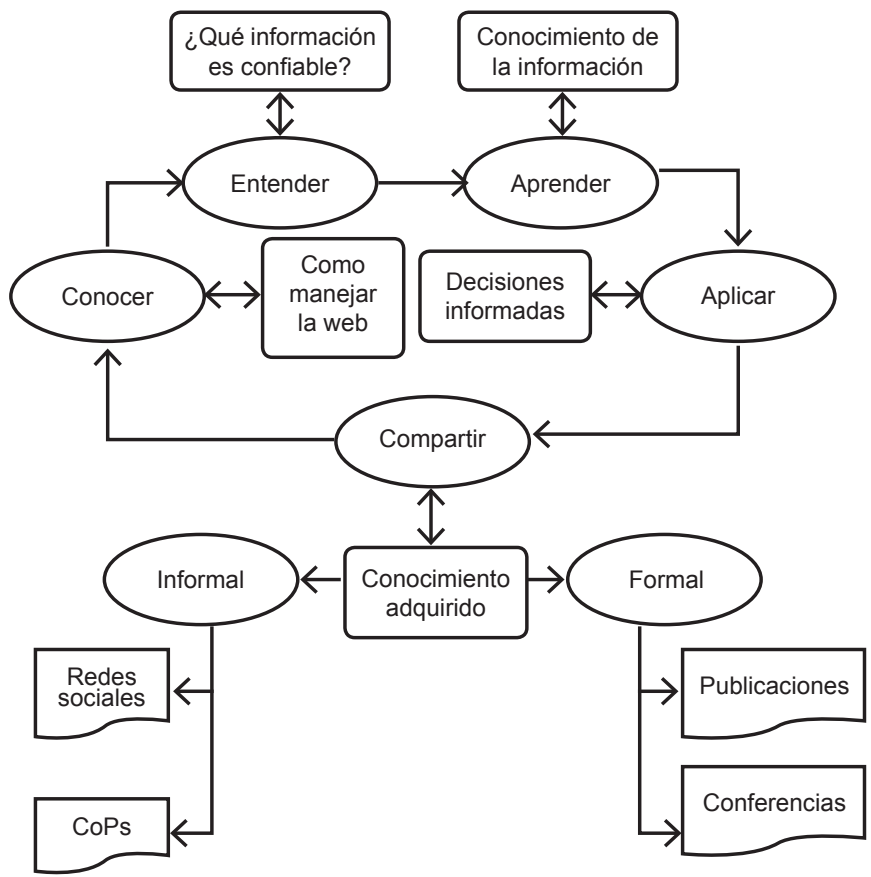

Figura 2. Ciclo de la responsabilidad del conocimiento. Fuente: http://marcelodagostino.wordpress.com

\section{CONCLUSIONES}

Tarde o temprano las sociedades, las instituciones y las personas estarán cada vez mas conectadas y cada vez habrá mas información disponible en la web, por ello, una de las principales responsabilidades de las instituciones y personas, con conocimiento sobre cómo acceder a información en salud fiable, es la de fortalecer las capacidades individuales y colectivas de las personas para que se puedan desarrollar funcionalmente y con equidad en la sociedad de la información.

El manejo de las tecnologías de la información también desempeña una función clave en la ampliación de la cobertura de servicios de salud a zonas remotas o vulnerables en donde la introducción de la tecnología móvil ha sido clave para responder a las necesidades de salud de las poblaciones rurales 0 aisladas, principalmente en los casos de emergencias sanitarias y en situaciones de desastres.

Es posible revolucionar la práctica de la salud pública en las Américas a través de la implementación de herramientas basadas en web que permiten la extracción automatizada de grandes cantidades de datos masivos estructurados y no estructurados (datos, textos, tweets, mensajes, audios, videos) y que son generados por los comportamientos sociales. Este fenómeno es conocido en su sigla del idioma inglés como Big data y forma parte de uno de los principales desafíos y oportunidades del presente y futuro cercano.

La gestión del conocimiento y la información, al facilitar el acceso a las tecnologías y al conocimiento, han demostrado servir para mejorar la calidad de vida de las personas. Por ende, se trata de un requisito indispensable para apoyar los objetivos de desarrollo sostenible.

Finalmente, es fundamental trabajar en estos temas de manera interprogramática y multidisciplinaria para que exista convergencia en acciones e inversiones en salud que fortalezcan la estrategia para el acceso universal a la salud y la cobertura universal de salud ${ }^{(7)}$.

\section{REFERENCIAS BIBLIOGRÁFICAS}

1. Organización Panamericana de la Salud. Estrategia y plan de acción sobre Esalud, $51^{\circ}$ Consejo Directivo; $63^{\text {a }} \mathrm{Se}-$ sión del Comité Regional de la OMS para las Américas. 2011 Sep 26-30; Washington, DC: OPS; 2011.

2. Union Internacional de Telecomunicaciones. World Telecommunication/ ICT Development Report 2010, Monitoring the targets, a mid-term review. Geneva: ITU; 2010.

3. Marti MC, D'Agostino MJ, Veiga de Cabo J, Sanz-Valero J. Alfabetización digital: un peldaño hacia la sociedad de la información. Med Segur Trab. 2008; 54(210); 11-5.
4. Organización Panamericana de la Salud. Conversaciones sobre eSalud. Washington, DC: OPS; 2014.

5. World Health Organization, International Telecomunication Union. National eHealth strategy toolkit. Geneva: WHO; 2012.

6. Organización Panamericana de la Salud. Agenda de Salud para las Américas 2008-2017 [Internet]. Washington, DC: OPS; 2007 [citado el 12 de marzo de 2011]. Disponible en: http:// new.paho.org/col/index.php?option=com_content\&task=view\&id=203\&Itemid $=259$
7. Organización Panamericana de la Salud. Estrategia para el acceso universal a la salud y la cobertura universal de salud. $53^{\circ}$ Consejo Directivo; $66^{a}$ Sesión del Comité Regional de la OMS para las Américas. 2014; Sep-Oct 29-03; Washington, DC: OPS; 2014.

Correspondencia: Marcelo José D'Agostino Dirección: 525 23rdst NW Washington. DC 20037, EE. UU

Teléfono: 1.202.974.3133

Correoelectrónico:dagostim@paho.org 